\title{
Active control of sound radiation from a fluid-loaded rectangular uniform plate
}

\author{
Yi Gu and Chris R. Fuller \\ Department of Mechanical Engineering, Virginia Polytechnic Institute and State University, \\ Blacksburg, Virginia 24061-0238
}

(Received 2 October 1991; revised 22 April 1992; accepted 4 August 1992)

Active control of sound radiation from a simply supported rectangular fluid-loaded plate is analytically studied. The plate is assumed to be excited by a point force at subsonic frequencies. The solution to the plate motion is based on the admissible functions for an in vacuo homogeneous plate, which is also the basis for Fourier decomposition of the fluid loading [B. E. Sandman, J. Acoust. Soc. Am. 61, 1502-1510 (1977) ]. Feed-forward control is carried out by using point forces applied to the plate. The amplitudes of the control forces are determined by the optimal solution of a quadratic cost function that integrates the far-field radiated acoustic pressure over a hemisphere in the radiation half-space. The results show that for subsonic disturbances, a high global reduction in radiated pressure is possible. For onresonant excitations, a reasonable sound reduction can be achieved with up to two properly located active control forces, and for off-resonant excitations, up to four control forces may be necessary. The results thus indicate that the active structural acoustic control approach will provide large attenuations in radiated sound when edge mode coupling induced by heavy fluid loading is present. The number and location of the control forces are determined so as to suppress the efficiently radiating modes. The far-field directivity pattern, the plate velocity autospectrum in the two-dimensional wave number domain, and the near-field pressure distribution are studied.

PACS numbers: 43.40.Vn, 43.40.Dx, 43.40.Rj

\section{INTRODUCTION}

There are many cases of practical interest to the industry and marine engineering in which the control of sound radiation from fluid-loaded plates is important. Much research has been done on the plate vibration response, the modal coupling effects due to the fluid loading, the radiation efficiency, etc. of fluid-loaded plates (Davies, 1977; Sandman, 1977; Lomas and Hayek, 1977; Fahy, 1985; Junger and Feit, 1986). All of the previous work is important in terms of understanding the behavior of sound radiation and dynamic structural response of fluid-loaded plates. On the other hand, active structural acoustic control (ASAC) has been applied recently to many structures such as plates (Fuller, 1988; Fuller et al., 1990a, 1991) and cylinders (Fuller and Jones, 1987; Fuller et al., 1990b) with light fluid loading (i.e., no radiation coupling) as well as to an infinite fluidloaded plate with discontinuities (Gu and Fuller, 1991, 1992).

The present study is focused on ASAC applied to a simply supported rectangular plate located in an infinite baffle with heavy fluid loading on one side, as shown in Fig. 1. The disturbance is a point force operating at a steady single frequency while control is achieved by point forces applied to the plate. The control objective is to minimize the total radiated power that is a quadratic function of the control force amplitudes. The study focuses on the behavior of the nearand far-field sound radiation as well as the wave-number distribution of the uncontrolled and controlled plate. The investigation is novel because it introduces for the first time the influence of edge mode coupling due to the heavy fluid loading into the ASAC technique. It was not known or un- derstood prior to this work how the modal coupling will affect control performance.

\section{ANALYSIS}

\section{A. Plate motion analysis}

In thin plate theory, the governing equation for the transverse deflection of the plate is

$$
D \nabla^{4} w+\rho_{p} h \frac{\partial^{2} w}{\partial t^{2}}=-q(x, y, t)-p_{0}(x, y, t),
$$

where $D=E h^{3} / 12\left(1-v^{2}\right)$ is the flexural rigidity of the plate, with $v$ denoting the Poisson ratio, $E$ is the plate material modulus of elasticity, $h$ is the thickness, $\rho_{p}$ is the mass density, $w(x, y, t)$ is the displacement of point $(x, y)$ at time $t$, $q(x, y, t)$ is the directly applied external force, which in this case includes the point disturbance force and the control forces, and $p_{0}(x, y, t)$ is the fluid loading pressure. Combining the wave equation, the Euler equation, and the boundary conditions on the plate and off the plate (Sandman, 1977), the modal amplitudes of the plate vibration can be solved from a complex nondiagonal matrix equation that reveals the coupled fluid-loading effects. The solution of Eq. (1) yields the fluid-loaded plate response $w(x, y, t)$ and the corresponding pressure field $p(x, y, z, t)$. The detailed procedure was described by previous researchers (Davies, 1971; Sandman, 1977; Lomas and Hayek, 1977), and the results are summarized here. The steady-state acoustic pressure is 


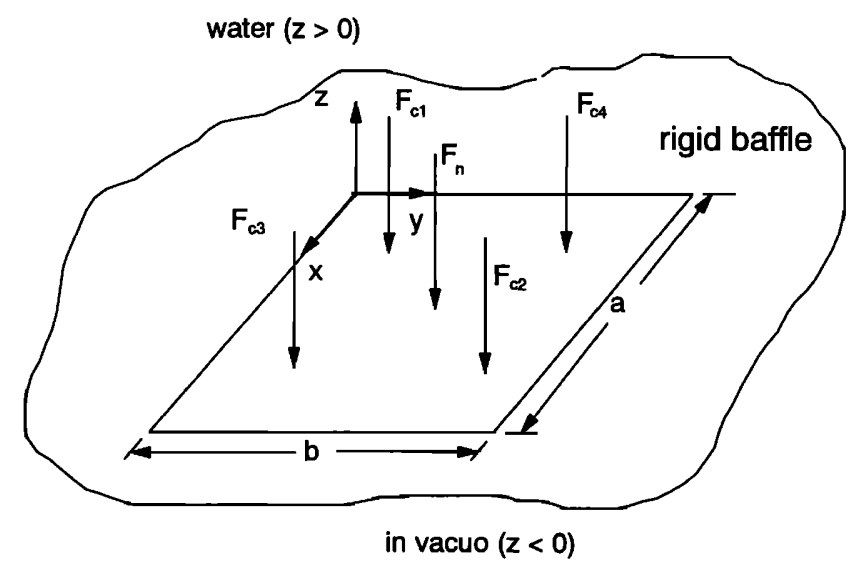

FIG. 1. System arrangement of a simply supported fluid-loaded plate.

$$
\begin{aligned}
p(x, y, z)= & 12 \frac{\rho_{f} c_{f}}{\rho_{p} c_{p}}\left(\frac{a}{h}\right)^{3}(i \omega) \frac{i k}{2 \pi} \\
& \times \sum_{m=1}^{\infty} \sum_{n=1}^{\infty} W_{m n} \int_{0}^{b} \int_{0}^{a} \sin \frac{m \pi x_{1}}{a} \\
& \times \sin \frac{n \pi y_{1}}{b} \frac{e^{-i k R}}{R} d x_{1} d y_{1},
\end{aligned}
$$

where $\rho_{f}$ and $c_{f}$ are the fluid density and the sound speed, respectively, $c_{p}$ is the flexural wave speed, $a$ and $b$ are the plate dimension in the $x$ and $y$ direction, $R=\sqrt{\left(x-x_{1}\right)^{2}+\left(y-y_{1}\right)^{2}+z^{2}}, \omega$ is the nondimensional excitation frequency, and $k$ is the acoustic wave number. Here, $W_{m n}$ are the modal coefficients that can be solved from the coupled matrix equation of

$$
\sum_{m=1}^{\infty} \sum_{n=1}^{\infty}\left(K_{r s m n}^{p}+i \omega R_{r s m n}^{f}-M_{r s m n}^{f+p}\right) W_{m n}=a_{r s},
$$

where $K_{r s m n}^{p}$ is the plate stiffness matrix, $\left(R_{r s m n}^{p}\right.$ $\left.+i \omega M_{r s m n}^{f+p}\right)$ is the fluid radiation resistance and combined mass matrix, and $a_{r s}$ are the modal components of the forcing function $q(x, y, t)$, respectively. The detailed definitions of these terms are given by Sandman (1977). To solve Eq. (3), the authors used a truncation of modes of $m=6, n=6$ for numerical estimation, since the difference is within $1 \%$ compared to that achieved by using a truncation of $m=10$, $n=10$.

For estimating the radiation pressure, the far-field pressure can be evaluated with the Rayleigh-Ritz method (Sandman, 1977) as given by

$$
p_{\mathrm{far}}(R, \theta, \phi)=\frac{6 D a \rho_{f} \omega^{3}}{\pi R \rho_{p} h^{3}} \sum_{m=1}^{\infty} \sum_{n=1}^{\infty} W_{m n} T_{m n}(\theta, \phi),
$$

in which

$$
\begin{aligned}
T_{m n}(\theta, \phi)= & \left(\frac{b}{a}\right)(m \pi)(n \pi) \\
& \times \frac{\left[1-(-1)^{m} e^{i K_{x}}\right]\left[1-(-1)^{n} e^{i K_{y}}\right]}{\left[(m \pi)^{2}-K_{x}^{2}\right]\left[(n \pi)^{2}-K_{y}^{2}\right]}
\end{aligned}
$$

where $K_{x}=k a \sin \theta \cos \phi$ and $K_{y}=k b \sin \theta \sin \phi$ are the definitions of the far-field radiation position, and $\theta$ and $\phi$ are defined in Fig. 2.

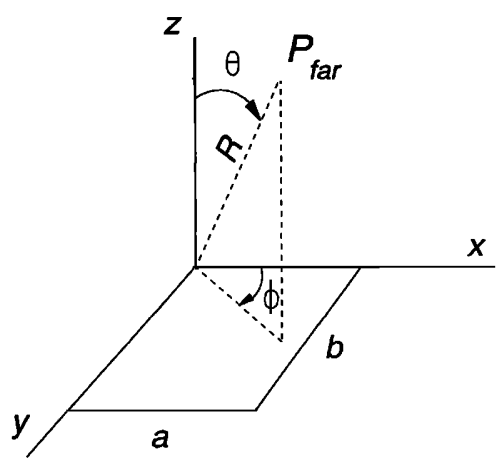

FIG. 2. Spatial coordinate definition.

\section{B. Sound radiation}

The total far-field radiated pressure due to the disturbance input and control forces is

$$
\begin{aligned}
p_{\mathrm{far}}^{t}(R, \theta, \phi) & =p_{\mathrm{far}}^{n}(R, \theta, \phi)+p_{\mathrm{far}}^{c}(R, \theta, \phi) \\
& =\sum_{i=1}^{N_{s}} B_{i} q_{i}+\sum_{j=1}^{N_{c}} A_{j} p_{j} \\
& =\{B\}^{T}\{q\}+\{A\}^{T}\{p\},
\end{aligned}
$$

in which the total far-field pressure $p_{\mathrm{far}}^{t}$ is the sum of $p_{\mathrm{far}}^{n}$ due to $N_{s}$ disturbance forces and $p_{\mathrm{far}}^{c}$ due to $N_{c}$ control forces, while $\{q\}$ is the disturbance force amplitude vector, $\{p\}$ is the control force amplitude vector, $\{B\}$ is the distribution function for the disturbance, and $\{A\}$ is the distribution function for the control force, respectively. Note that both $\{A\}$ and $\{B\}$ are functions of $W_{m n}$, which are the modal amplitudes of the solution to Eq. (1). Combining Eq. (4) and $\mathrm{Eq} .(6)$ results in the following equations:

$$
A_{j}=\frac{6 a^{4} \rho_{f} \omega^{2}}{\pi R \rho_{p} h^{3}} \sum_{m=1}^{\infty} \sum_{n=1}^{\infty} \frac{W_{m n}}{p_{j}} T_{m n}(\theta, \phi)
$$

and

$$
B_{i}=\frac{6 a^{4} \rho_{f} \omega^{2}}{\pi R \rho_{p} h^{3}} \sum_{m=1}^{\infty} \sum_{n=1}^{\infty} \frac{W_{m n}}{q_{i}} T_{m n}(\theta, \phi),
$$

where $A_{j}$ and $B_{i}$ are the $j$ th element and the $i$ th element of vectors $\{A\}$ and $\{B\}$, respectively, and $p_{j}$ and $q_{i}$ are the $j$ th element and the $i$ th element of the control force vector $\{p\}$ and the disturbance forcing vector $\{q\}$, respectively.

\section{Optimal control}

The objective of the optimal control is to minimize the far-field sound radiation over a hemisphere above the plate in the fluid half-space. The cost function based on the farfield acoustic power is expressed as (Fuller, 1988)

$\Phi=\frac{1}{R^{2}} \int_{S}\left|p_{\mathrm{far}}^{t}\right|^{2} d s=\int_{0}^{2 \pi} \int_{0}^{\pi / 2}\left|p_{\mathrm{far}}^{t}\right|^{2} \sin \theta d \theta d \phi$,

which can be written in matrix form as

$$
\begin{aligned}
\Phi= & \{p\}^{T}[A]\{p\}^{*}+\{q\}^{T}[B]\{p\}^{*}+\{p\}^{T}[B]^{H}\{q\}^{*} \\
& +\{q\}^{T}[C]\{q\}^{*},
\end{aligned}
$$


where superscript " $T$ " denotes transposition, “*” denotes conjugation, and " $H$ " denotes transposition and conjugation. Matrices $[A],[B]$, and $[C]$ are the results of substituting the vector sum of Eq. (6) into Eq. (9) and can be expressed as follows:

$$
\begin{aligned}
& {[A]_{N_{c} \times N_{c}}=\int_{0}^{2 \pi} \int_{0}^{\pi / 2}\left[\{A\}\{A\}^{H}\right] \sin \theta d \theta d \phi,} \\
& {[B]_{N_{s} \times N_{c}}=\int_{0}^{2 \pi} \int_{0}^{\pi / 2}\left[\{B\}\{A\}^{H}\right] \sin \theta d \theta d \phi,}
\end{aligned}
$$

and

$$
[C]_{N_{s} \times N_{s}}=\int_{0}^{2 \pi} \int_{0}^{\pi / 2}\left[\{B\}\{B\}^{H}\right] \sin \theta d \theta d \phi .
$$

In order to minimize the acoustic power expressed in Eq. (9), the cost function is differentiated with respect to the control force amplitude $\{p\}$ and set to zero, as outlined by Nelson et al. (1987) and Lester and Fuller (1990). As the optimal solution for the minimization of the cost function defined in Eq. (9), the control force amplitude is

$$
\{p\}=-[A]^{-1}[B]^{T}\{q\} .
$$

Equation (14) relates the control force amplitude $\{p\}$ with the disturbance amplitude $\{q\}$. The relationship implies that, given the disturbance, the active feedforward control can be implemented by proper amplitude and phase adjustments of the control forces through Eq. (14) to minimize the sound radiation in the far field. Here, $[A]$ is the distribution matrix of control forces and $[B]$ is the distribution matrix relating the coupling between the control forces and the disturbance.

\section{RESULTS AND DISCUSSION}

The numerical evaluation is based on an aluminum rectangular plate of which the material properties and dimensions are listed in Table I. The center-point-driven response of the plate depicted in Fig. 3 illustrates the plate resonances with or without heavy fluid loading. For the problem considered here, the excitation frequencies are only those that dominantly excite the low-order modes of the plate and are well below the coincidence frequency, $f_{c}=c_{f}^{2}\left(m_{p} / D\right)^{1 / 2}$ $=23966 \mathrm{~Hz}$ for the given plate, where $c_{f}$ is the sound velocity in the sea water and $m_{p}$ the plate density per area. The disturbance force amplitude is taken as $10 \mathrm{~N}$ for all the cases calculated in the following examples.

The presence of fluid loading lowers the resonant frequencies of the plate response but does not significantly

\begin{tabular}{|c|c|c|c|c|}
\hline System & $\begin{array}{l}\text { Phase } \\
\text { speed } \\
(\mathrm{m} / \mathrm{s})\end{array}$ & $\begin{array}{c}\text { Density } \\
\left(\mathrm{kg} / \mathrm{m}^{3}\right)\end{array}$ & $\begin{array}{l}\text { Thickness } \\
(\mathrm{m})\end{array}$ & $\begin{array}{l}\text { Size } \\
(\mathrm{m})\end{array}$ \\
\hline $\begin{array}{l}\text { Aluminum plate } \\
\text { Seawater }\end{array}$ & $\begin{array}{l}5432 \\
1500\end{array}$ & $\begin{array}{l}2700 \\
1026\end{array}$ & $\begin{array}{c}0.009525 \\
\ldots\end{array}$ & $\begin{array}{c}0.5588 \times 0.8636 \\
\ldots\end{array}$ \\
\hline
\end{tabular}
change the structural mode shapes (Fahy, 1985). The natu-

TABLE I. Material properties of an aluminum rectangular plate and fluid medium.

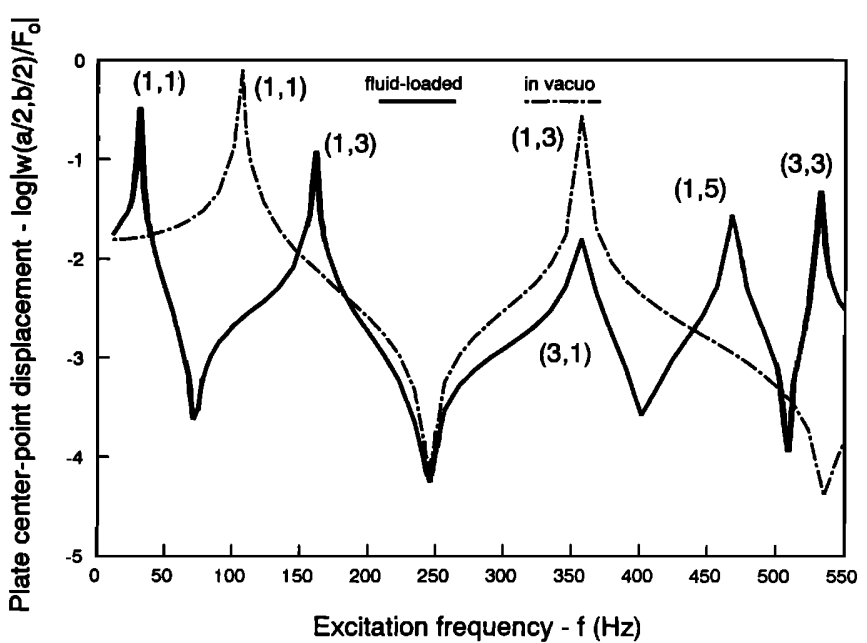

FIG. 3. Frequency response of a rectangular plate.

ral frequencies of the first several modes were estimated numerically from the plate displacement response of Fig. 3 and compared to those results calculated with the approximate expression provided by Fahy (1985). The far-field pressure was calculated using Eq. (6) in which the optimal control forces were based on Eq. (14) for the controlled case. With a harmonic point force applied at the center of the plate as the disturbance, the plate is excited at on- and off-resonant frequencies. The near-field pressure distributions illustrate how the control forces modify the sound radiation sources and change the nature of structural acoustic coupling near the surface of the plate.

The two-dimensional wave-number domain $\left(k_{x}, k_{y}\right)$ analysis demonstrates the change within the supersonic wave-number region $\left(\sqrt{k_{x}^{2}+k_{y}^{2}} \leqslant \omega / c\right)$. The wave-number spectrum of the plate velocity is given by the Fourier transform of the plate velocity as follows:

$$
\begin{aligned}
\widetilde{V}\left(k_{x}, k_{y}\right)= & \int_{-\infty}^{\infty} \int_{-\infty}^{\infty} v(x, y) e^{-i\left(k_{x} x+k_{y} y\right)} d x d y \\
= & i \omega c_{p} \sum_{m=1}^{\infty} \sum_{n=1}^{\infty} W_{m n} \\
& \times\left(\frac{m \pi}{\left.k_{x}^{2}-(m \pi) / a\right)^{2}}\left[(-1)^{m} e^{-i k_{x} a}-1\right]\right) \\
& \times\left(\frac{(n \pi / b)}{k_{y}^{2}-(n \pi / b)^{2}}\left[(-1)^{n} e^{-i k_{y} b}-1\right]\right),
\end{aligned}
$$

where $v(x, y)=i \omega w(x, y)$ and $w(x, y)$ is the solution of Eq. (1). The velocity autospectrum is used to evaluate the wavenumber domain energy and its expression is

$$
\left|\widetilde{V}\left(k_{x}, k_{y}\right)\right|^{2}=\widetilde{V}\left(k_{x}, k_{y}\right) \widetilde{V}^{*}\left(k_{x}, k_{y}\right)
$$

It should be noted that the velocity wave-number autospectrum is also a function of the excitation frequency. 


\section{A. Resonant frequencies}

For a simply supported rectangular plate in vacuo, the natural frequencies are estimated as

$$
\omega_{m n}=\sqrt{\frac{D}{m_{p}}}\left[\left(\frac{m \pi}{a}\right)^{2}+\left(\frac{n \pi}{b}\right)^{2}\right],
$$

where $m_{p}=\rho_{p} h$. For a rectangular plate submerged in heavy fluid, it is assumed that the natural frequencies fall below their in vacuo values in proportion to the square root of the ratio of the loaded to unloaded modal masses (Fahy, 1985). According to Fahy, the analysis of reactive loading on structural waves having wave numbers much greater than an acoustic wave number has shown that the effective added mass per unit area is $\rho_{f} / k_{m n}$, where $\rho_{f}$ is the fluid density and $k_{m n}$ is the primary effective wave-number component of the vibration. The approximate expression of the fluid-loaded structure natural frequency is (Fahy, 1985)

$$
\omega_{m n}^{\prime} \approx \omega_{m n}\left[1+\left(\rho_{f} / m_{p} k_{m n}\right)\right]^{-1 / 2},
$$

where $\omega_{m n}$ is the corresponding in vacuo natural frequency defined by Eq. (17) and $k_{m n}=\sqrt{(m \pi / a)^{2}+(n \pi / b)^{2}}$.

Hence, there are two approximate methods to determine fluid-loaded plate natural frequencies: one is to observe the peak values from the frequency response magnitudes such as from Fig. 3, since the nonlinearity of Eq. (3) makes an explicit solution of eigenvalues unavailable. The other is to use Eq. (18). In the following discussion, the results obtained with these two different methods are compared and found to be very consistent in most cases.

Figure 3 illustrates the center-point displacement magnitude of the plate for center-point excitation. The results are very similar to those previously estimated by Sandman (1977). Because of the location of the drive point, it is seen that even numbered modes can not be excited, so that only odd-odd modes appear on the response diagram. The in vacuo resonances are well predicted by Eq. (17). The natural frequencies of the fluid-loaded case evaluated by two different approaches also converge well (referring to Tables II and III). The relative errors between the results are reasonable $\left(8.0 \%\right.$ for $\omega_{13}^{\prime}, 9.1 \%$ for $\omega_{31}^{\prime}, 4.6 \%$ for $\omega_{15}^{\prime}$, and $4.3 \%$ for $\omega_{33}^{\prime}$ ) except for the first mode ( $30 \%$ for $\omega_{11}^{\prime}$ ). The comparison of these results suggests that in general Eq. (18) is a fairly good estimate for fluid-loaded plate lower-order modal natural frequencies when $k_{m n} \gg k$ and the discrepancy in estimating the first mode is noted.

\section{B. Fundamental mode excitation}

When the disturbance frequency coincides with the first mode $(1,1)$ resonance, a relatively high sound radiation arises. Because of the location and the frequency of the exci-

TABLE II. Natural frequencies $(\mathrm{Hz})$ of the fluid-loaded plate estimated with Fahy's approximate formula (Fahy, 1985).

\begin{tabular}{lccc}
\hline \hline Mode $(m, n)$ & 1 & 3 & 5 \\
\hline 1 & 40.41 & 173.8 & 489.7 \\
3 & 388.9 & 555.2 & 905.7 \\
\hline \hline
\end{tabular}

TABLE III. Natural frequencies $(\mathrm{Hz})$ of the fluid-loaded plate estimated from numerical frequency response evaluation.

\begin{tabular}{lccc}
\hline \hline Mode $(m, n)$ & 1 & 3 & 5 \\
\hline 1 & 30.94 & 160.9 & 467.8 \\
3 & 356.4 & 532.2 & $\cdots$ \\
\hline \hline
\end{tabular}

tation, the fundamental mode dominates the plate vibration and the sound radiation. Since the plate is vibrating in a single efficiently radiating monopole mode, the task is to try to suppress this mode by secondary forces in order to reduce the sound radiation. With one control force located at $\left(x_{1}, y_{1}\right)=(a / 4, b / 4)$, the controlled plate is seen to radiate like a dipole (referring to Fig. 4 ) and the sound radiation is attenuated in the far field by around 65 to $85 \mathrm{~dB}$. From the near-field pressure distribution shown in Fig. 5(a) and (b) it is illustrated that the overall pressure level decreases about $44 \mathrm{~dB}$ near the surface of the plate. This indicates that the suppression of the $(1,1)$ mode leads to a global sound reduction. With two control forces located at $\left(x_{1}, y_{1}\right)=(a / 4, b / 4)$ and $\left(x_{2}, y_{2}\right)=(3 a / 4,3 b / 4)$, a further 25 to $30 \mathrm{~dB}$ of far-field pressure attenuation is achieved as observed in Fig. 4. Comparing Fig. 5(b) and Fig. 5(c) reveals that not only a further 15 to $20 \mathrm{~dB}$ reduction of pressure level is achieved in the near field, but the pattern of the radiation source is also changed from a two-lobe type to a three-lobe type. This implies that not only the dominant $(1,1)$ mode is suppressed, but the relations between the residual modes are re-adjusted so as to make their overall contribution to sound radiation less efficient. This phenomenon is known as "modal restructuring". When four control forces are located at one-sixth of the lengths away from the plate edges, even further sound attenuation is observed in the far field (the residual pressure directivity is localized around the origin in Fig. 4). The force amplitudes and locations are listed in Table IV.

The near-field sound pressure magnitude distribution shown in Fig. 5(d) indicates that further modal restructur-

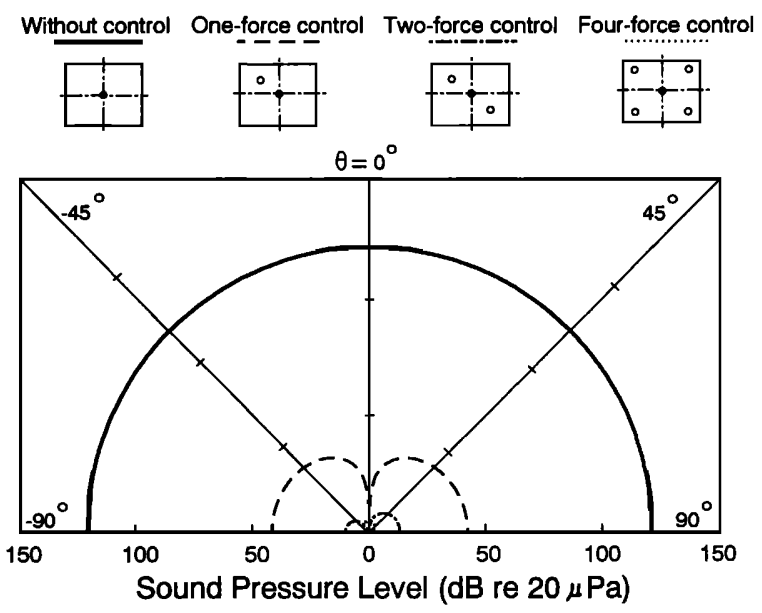

FIG. 4. Far-field directivity pattern: on-resonance excitation, $f=31 \mathrm{~Hz}$, $\phi=0$.

Y. Gu and C. R. Fuller: Active control of sound radiation 


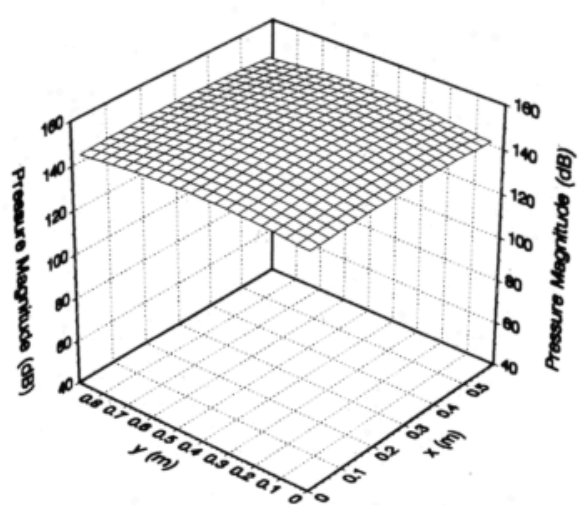

(a) Disturbance only

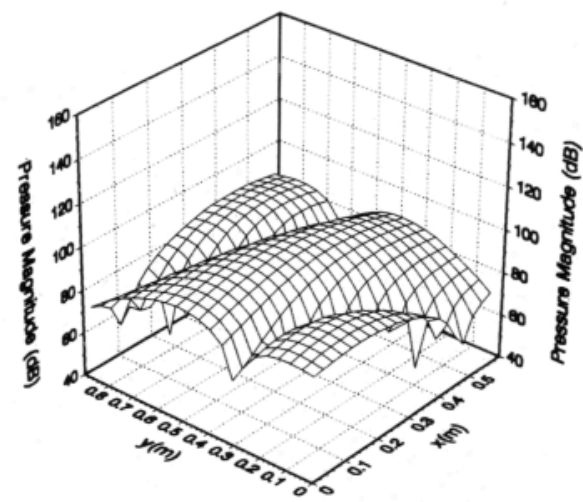

(c) With two control forces

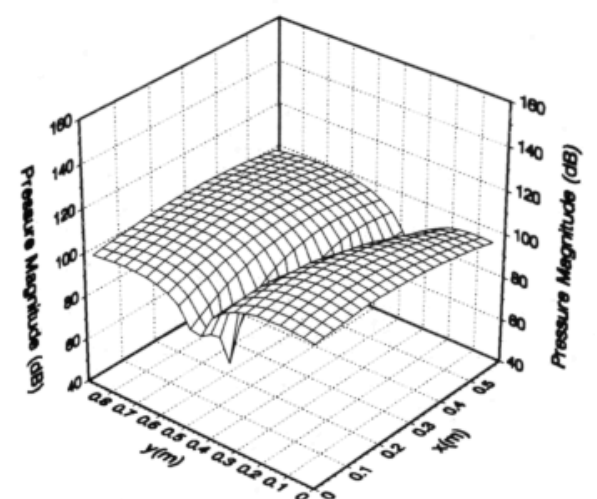

(b) With one control force

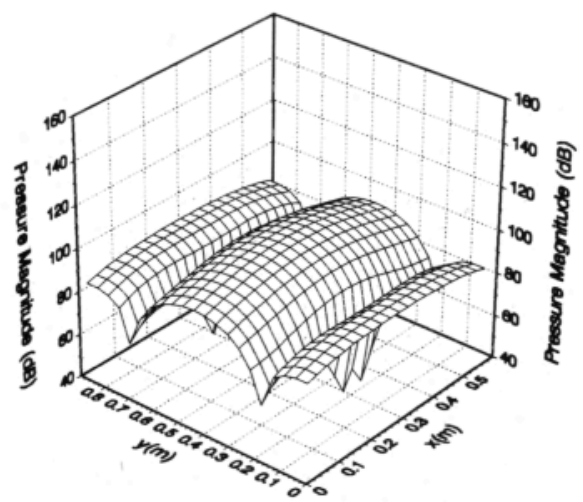

(d) With four control forces

FIG. 5. Near-field sound pressure level at $z=0.01 \mathrm{~m}, f=31 \mathrm{~Hz}$.

ing is performed since the overall pressure level does not seem to be lower than that shown in Fig. 5(c), but different higher-order source patterns are observed in these two controlled cases. Comparing the controlled residual pressure distributions shown in Fig. 5(b), (c), and (d), to the uncon-

TABLE IV. Disturbance and control force amplitudes and locations, $a-$ plate length in the $x$ direction, $b$-plate length in the $y$ direction.

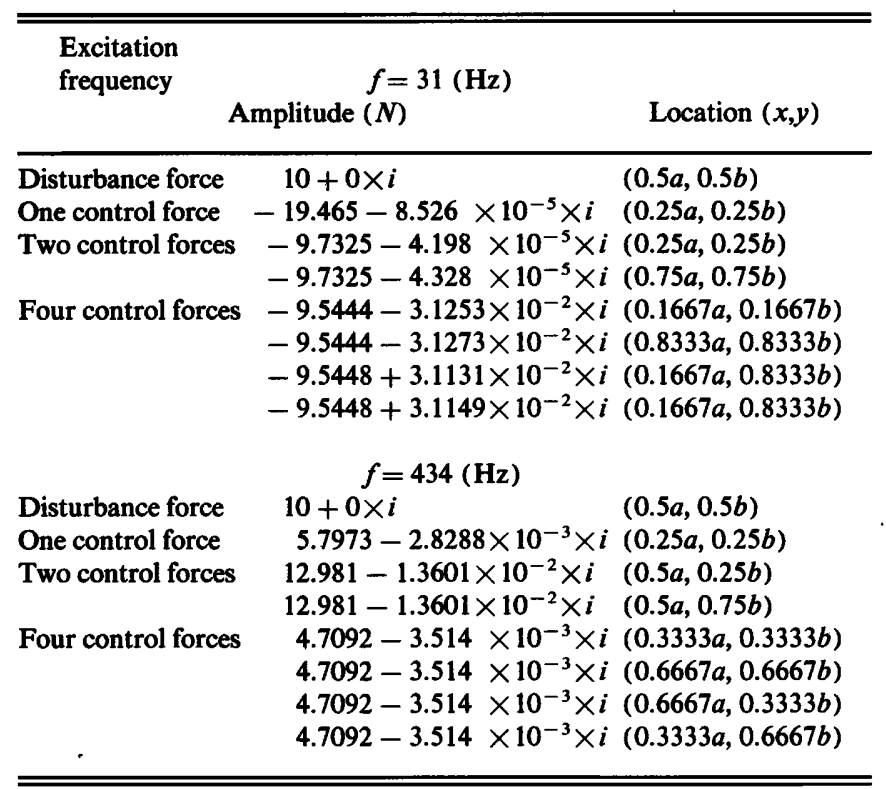

trolled pressure distribution shown in Fig. 5(a), it is noted that the modal suppression, i.e., the suppression of the efficient $(1,1)$ mode, is the dominant cause of the sound reduction. This observation is extendible to those cases when only one efficient mode is dominantly excited to radiate sound.

To better explain the sound power reduction, the plate velocity autospectrum in a two-dimensional wave-number domain is calculated and the results are shown in Fig. 6. (The reference value in Figs. 6 and 9 is arbitrarily taken as $6.36 \times 10^{-9} \mathrm{~m}^{2} / \mathrm{s}^{2}$ so that the autospectrum represents relative values.) It is observed that the supersonic region of the wave-number spectrum, illustrated by the area within the small circle where $\sqrt{k_{x}^{2}+k_{y}^{2}} \leqslant k$, decreases with the increase of number of control forces. This clearly explains that the active control reduces the sound radiation energy through reducing the radiated power in the supersonic region. In addition, it is observed that the reduction in sound radiation is not necessarily accompanied by reduction in plate vibration. For example, the area outside the supersonic region remains almost the same level in Fig. 6(c) and (d), while the supersonic region is reduced. The corresponding far-field pressures demonstrate a fall in magnitude (refer to Fig. 4). The results illustrate the important observation that only the wave-number spectrum within the supersonic region is relevant to the far-field sound radiation and hence reduction. On the other hand, the modal suppression is also confirmed by comparing Fig. 6(b), (c), and (d) with Fig. 6(a), respectively, the velocity autospectrum is reduced within and outside the supersonic region. This indicates that 


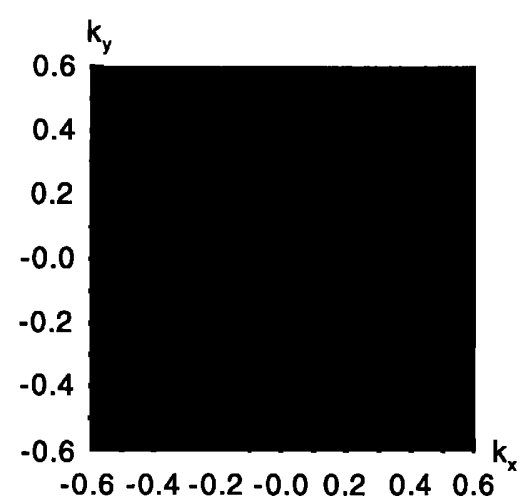

(a) Disturbance only

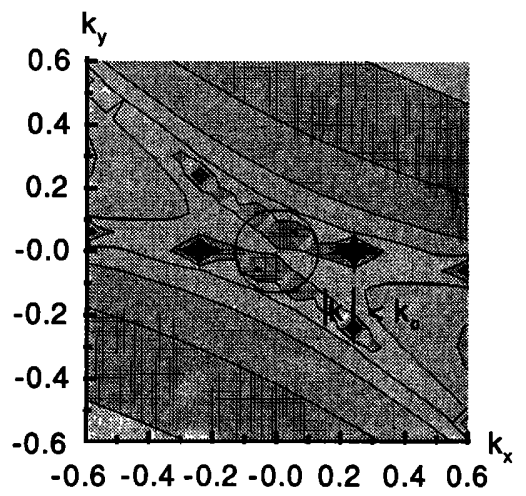

(c) With two control forces

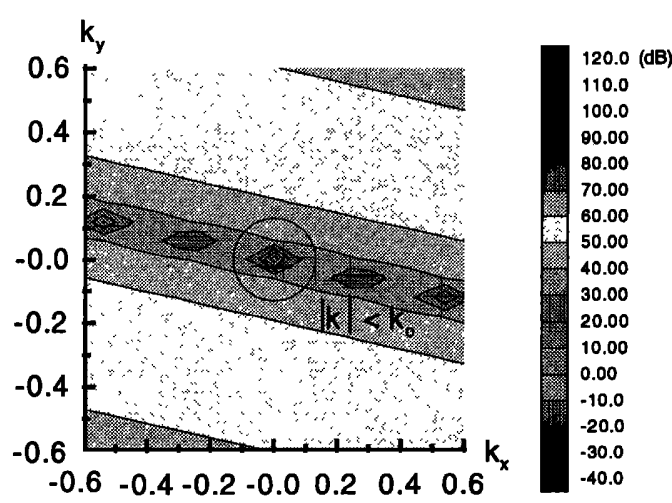

(b) With one control force

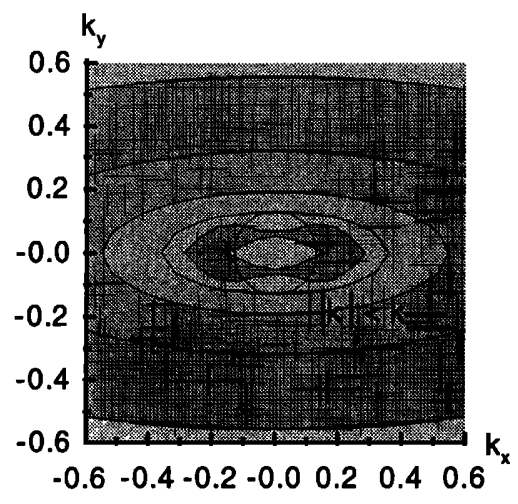

(d) With four control forces

FIG. 6. Wave-number domain plate velocity autospectrum, $f=31 \mathrm{~Hz}$.

there is not only a reduction in sound radiation level, but a reduction in plate vibration magnitude level as well.

It is also interesting to examine the optimal control force values that are given in Table IV. For the on-resonant case of $f=31 \mathrm{~Hz}$, it is apparent that the control forces are always nearly purely real and $180^{\circ}$ out-of-phase with the disturbance force. For a single control force, a much larger magnitude of optimal control force than disturbance is required, although this will depend upon location of the forces. When multiple control forces are used, the control force magnitudes are of the same order as the disturbance and largely independent of location. The results indicate that for the onresonant case, although multiple control inputs do not significantly increase the sound reduction, they have the advantage of keeping the control force magnitudes lower. This effect, however, needs further investigation before definite conclusions can be made.

\section{Off-resonant excitation}

The off-resonant example is illustrated with the plate centrally driven at frequency $f=434 \mathrm{~Hz}$. From Fig. 3 it can be seen that this frequency is higher than the resonance of mode $(3,1)$ so that more modes are involved in the plate response. The results show that, although the sound radiation level due to the disturbance is relatively lower than that of the on-resonant excitation example, reasonable sound reduction is much harder to obtain. In this case, the $(3,1)$, $(1,5)$, and $(3,3)$ modes combine to contribute to the plate vibration and sound radiation. It is thus difficult to position one control force to couple into all three modes, in such a way as to reduce their respective sound radition properly to achieve a global sound reduction. This is illustrated by the results of Fig. 7 where one control force only reduces the farfield radiation by around 2 to $4 \mathrm{~dB}$. When two or more control forces are employed, reasonable sound reduction is achieved. Figure 7 shows that about 15 to $30 \mathrm{~dB}$ of attenuation is obtained in the far field with two control forces and

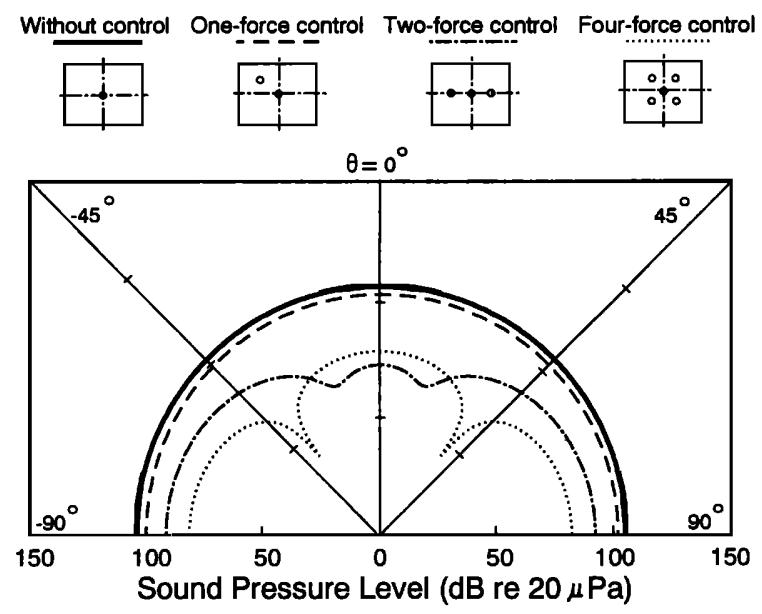

FIG. 7. Far-field directivity pattern: off-resonant excitation, $f=434 \mathrm{~Hz}$, $\phi=0$.

Y. Gu and C. R. Fuller: Active control of sound radiation 


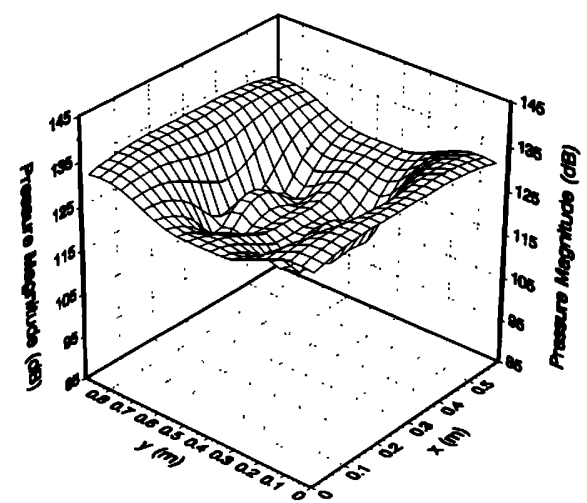

(a) Disturbance only

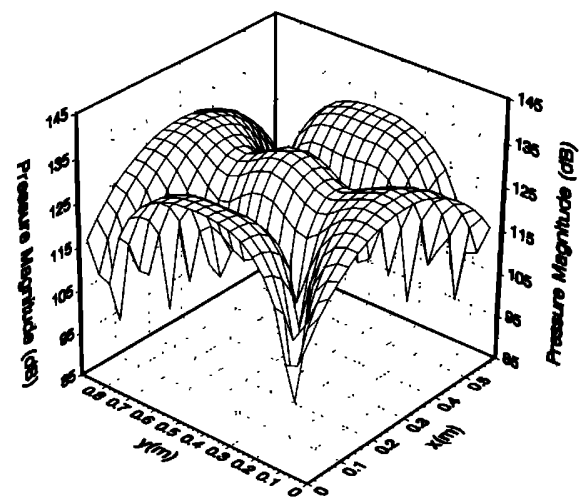

(c) With two control forces

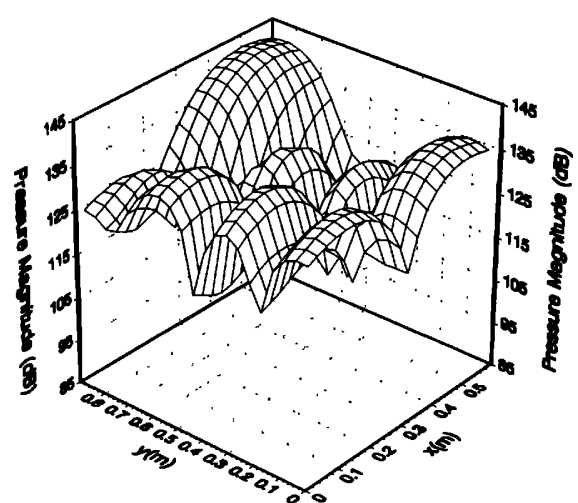

(b) With one control force

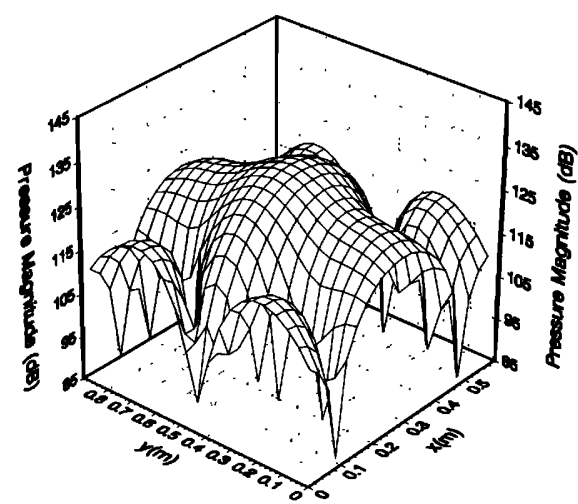

(d) With four control forces

FIG. 8. Near-field sound pressure level at $z=0.01 \mathrm{~m}, f=434 \mathrm{~Hz}$.

the directivity patterns suggest that the radiation source is of multipole type. Four control forces provide a further sound reduction in a global extent of about 10 to $20 \mathrm{~dB}$ although in the region from $\theta=-25^{\circ}$ to $\theta=25^{\circ}$ the radiation increases by about 10 to $15 \mathrm{~dB}$ over the two-control-force case.

An examination of near-field pressure distribution from Fig. 8(a) to (d) implies that the off-resonant plate source is far more complicated than the on-resonant case and a farfield sound reduction does not always accompany a significant overall pressure level reduction in the near field due to the "modal restructuring" phenomena discussed previously. The near-field pressure distribution in Fig. 8(a) is the result of radiation of the $(3,1),(1,5)$ and $(3,3)$ modes. When one control force is applied, there is no apparent reduction of the pressure level, as shown in Fig. 8(b), but there is some change in the shapes of the source pattern. Meanwhile it is seen in Fig. 9(b) that there is some minor radiation reduction, as illustrated by a decrease of the velocity autospectrum in the upper right area in the supersonic circle. This indicates that ASAC provides some attenuation, although the result is not as good as the on-resonant case when one control force is used. Another interesting phenomenon is that by observing the subsonic regions in Fig. 9(a) and (b), respectively, it is shown that the subsonic region in Fig. 9 (b) has a higher level than that in Fig. 9(a), which indicates that the plate vibration level may be higher, when control is applied (this can only be confirmed when the velocity autospectrum is plotted in the full range of $\left.-\infty<k_{x}<\infty,-\infty<k_{y}<\infty\right)$. This indicates two points: (1) ASAC does not always reduce the structural response; (2) modal restructuring sometimes can play an important role in reducing the sound radiation, particularly for off-resonant cases. When two control forces are applied, it is observed that the overall near-field pressure level is only slight reduced [Fig. 8(c) ], but a much better sound reduction is observed in the far field (Fig. 7) as well as in the supersonic region of velocity autospectrum [Fig. 9(c) ]. From the near-field pressure distribution shown in Fig. 8(c) it can be concluded that the increase of source order leads to the sound reduction. Finally when four control forces are applied on the plate, the overall near-field pressure level is reduced by about $10 \mathrm{~dB}$ and the source order is further modified [Fig. 8(d)]. A further reduction of velocity autospectrum in supersonic is also observed in Fig. 9(d) corresponding to the far-field pressure reduction in Fig. 7. In this four-force-control case, it can thus be concluded that modal suppression as well as modal restructuring is the mechanism of modifying the panel source and reducing the sound radiation. The drop in plate response for the case of Fig. 8(d) is due to the number of control forces being of the order of significant plate modes in terms of sound radiation.

Table IV provides the optimal control force magnitudes for the off-resonant case of $f=434 \mathrm{~Hz}$. The control forces are again nearly purely real but are now in phase with the disturbance in contrast to the on-resonant case. When one control force is used, the force magnitude is of the order of the disturbance, but corresponding sound power reduction is small. Using two control forces leads to better sound re- 


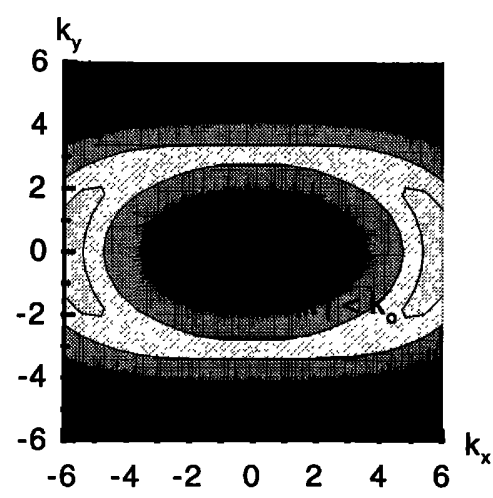

(a) Disturbance only

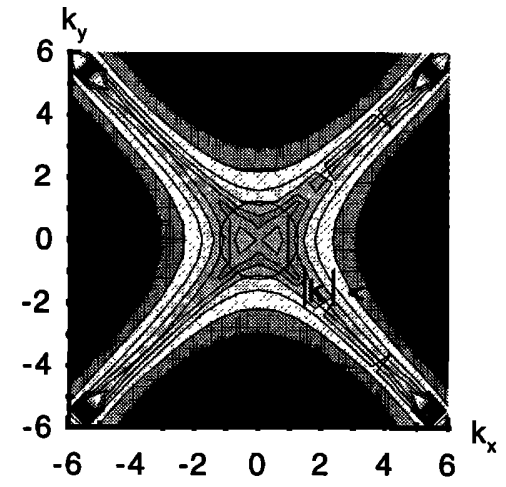

(c) With two control forces

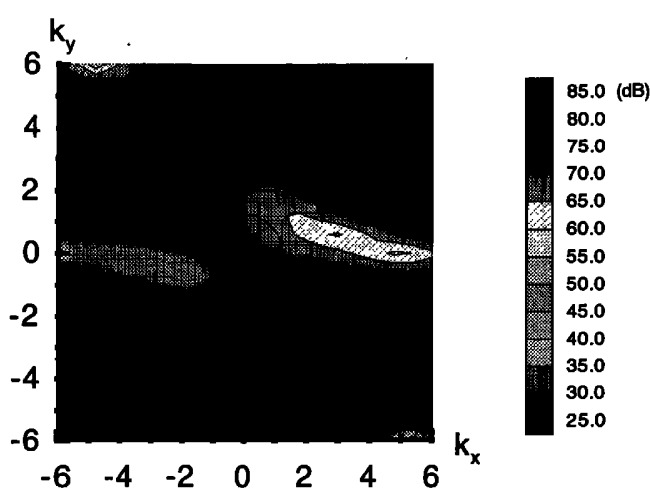

(b) With one control force

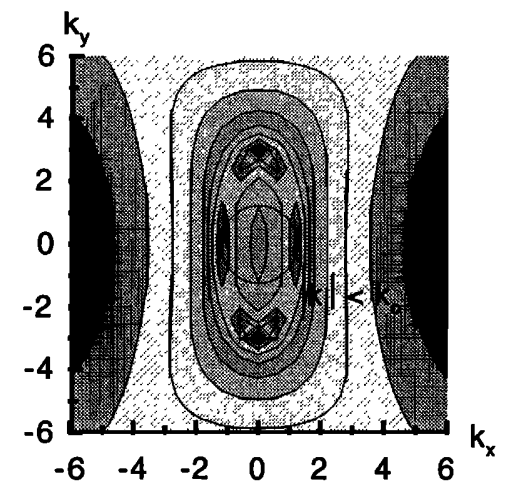

(d) With four control forces

FIG. 9. Wave-number domain plate velocity autospectrum, $f=434 \mathrm{~Hz}$.

duction (see Fig. 7) and the control magnitudes are also of the order of the disturbance. Four control forces leads to high sound power reduction and the control magnitudes are now reduced, in line with the on-resonant case.

\section{CONCLUDING REMARKS}

Active control of sound radiation from a fluid-loaded rectangular plate excited by a centrally located point force at subsonic frequencies has been analytically studied. The control forces are chosen so as to minimize the total acoustic power radiated into a hemisphere in the fluid-loaded halfspace. The reduction in sound radiation has been shown to depend on the excitation frequency that determines which modes will contribute dominantly to the total radiation. In general, off-resonant excitations are more difficult to control than on-resonant ones since more modes are involved. In the cases studied in this paper, up to two control forces are needed to control radiation for on-resonant excitation and up to four control forces for off-resonant excitation.

A two-dimensional wave-number domain analysis of the plate response illustrates how the wave-number components in the supersonic region decrease when active control is applied. This approach reveals the cause of sound reduction from the point of view of plate vibration radiating components. It is demonstrated that for plates with heavy fluid loading, sound radiation control occurs by two major mechanisms viz. (1) modal suppression in which dominantly radiating modes are controlled in magnitude and (2) modal restructuring in which the plate averaged response is little changed but has a lower radiation efficiency due to a more complex residual shape. The sound reduction achieved in on-resonant case is mainly through modal suppression, and the sound reduction achieved in off-resonant case is achieved primarily through modal restructuring.

This study adds new understanding to research in controlling the sound radiation from finite fluid-loaded plates. The results indicate that the ASAC feedforward control approach will provide high sound attenuation for vibrating structures submerged in heavy fluids including edge radiation coupling phenomena. Future work will study the effect of localized structural discontinuities as well as experimentally confirm the above conclusions.

\section{ACKNOWLEDGMENT}

The authors wish to acknowledge the support of this work to the sponsor, the Office of Naval Research, under Grant No. ONR-N00014-92-j-1170.

Davies, H. G. (1971). "Low frequency random excitation of water-loaded rectangular plates," J. Sound Vib. 15, 107-126.

Fahy, F. (1985). Sound and Structural Vibration: Radiation, Transmission and Response (Academic, Orlando, FL), pp. 113-142.

Fuller, C. R., and Jones, J. D. (1987). "Experiments on reduction of propeller induced interior noise by active control of cylinder vibration," $J$. Sound Vib. 112, 389-395. 
Fuller, C. R. (1988). "Active sound transmission/radiation from elastic plates by vibration inputs. I. Analysis," Proceedings Internoise '88, Avignon, France (Noise Control Foundation, Poughkeepsie, NY), pp. 10611064.

Fuller, C. R. (1990a). "Active control of sound transmission/radiation from elastic plates by vibration inputs. I. Analysis," J. Sound Vib. 136, 115.

Fuller, C. R., Snyder, S. D., Hansen, C. H., and Silcox, R. J. (1990b). “Active control of interior noise in model aircraft fuselages using piezoceramic actuators," AIAA Paper 90-3922.

Fuller, C. R., Hansen, C. H., and Snyder, S. D. (1991). "Experiments on active control of sound radiation from a panel using a piezoceramic actuator," J. Sound Vib. 150, 179-190.

Gu, Y., and Fuller, C. R. (1991). "Active control of sound radiation due to subsonic wave scattering from discontinuities on fluid loaded plates. I:
Far-field pressure," J. Acoust. Soc. Am. 90, 2020-2026.

Gu, Y., and Fuller, C. R. (1992). "Active control of sound radiation due to subsonic wave scattering from discontinuities on fluid loaded plates. II: Plate vibration" (in preparation).

Junger, M. C., and Feit, D. (1986). Sound, Structures, and Their Interaction (MIT, Cambridge), pp. 235-257.

Lester, H. C., and Fuller, C. R. (1990). "Active control of propeller-induced noise fields inside a flexible cylinder," AIAA J. 28 (8), 1364-1380.

Lomas, N. S., and Hayek, S. I. (1977). "Vibration and acoustic radiation of elastically supported rectangular plates," J. Sound Vib. 52, 1-25.

Nelson, P. A., Curtis, A. R. D., Elliott, S. J. and Bullmore, A. J. (1987). "The Minimum Power Output of Free Field Point Sources and the Active Control of Sound," J. Sound Vib. 116, 397-414.

Sandman, B. E. (1977). "Fluid-loaded vibration of an elastic plate carrying a concentrated mass," J. Acoust. Soc. Am. 61, 1502-1510. 